\title{
GLOBAL SOLUTIONS, STRUCTURE OF INITIAL DATA AND THE NAVIER-STOKES EQUATIONS
}

\author{
PIOTR BOGUSŁAW MUCHA \\ Institute of Applied Mathematics and Mechanics, Warsaw University \\ Banacha 2, 02-097 Warszawa, Poland \\ E-mail: p.mucha@mimuw.edu.pl
}

\begin{abstract}
In this note we present a proof of existence of global in time regular (unique) solutions to the Navier-Stokes equations in an arbitrary three dimensional domain with a general boundary condition. The only restriction is that the $L_{2}$-norm of the initial datum is required to be sufficiently small. The magnitude of the rest of the norm is not restricted. Our considerations show the essential role played by the energy bound in proving global in time results for the Navier-Stokes equations.
\end{abstract}

1. Introduction. The subject of this note is the existence of global in time regular solutions to the evolutionary Navier-Stokes equations in a rigid three dimensional domain. We concentrate our attention on the initial value problem

$$
\begin{array}{ll}
v_{t}+v \cdot \nabla v-\nu \Delta v+\nabla p=0 & \text { in } \Omega \times(0, T), \\
\operatorname{div} v=0 & \text { in } \Omega \times(0, T), \\
\left.v\right|_{t=0}=v_{0} & \text { on } \Omega,
\end{array}
$$

where $v=\left(v^{1}, v^{2}, v^{3}\right)$ represents the sought velocity field, $p$ is the sought pressure, $\nu$ is the constant positive viscous coefficient and $v_{0}$ the initial velocity which is required to satisfy the compatibility condition $\operatorname{div} v_{0}=0$. We do not restrict our attention only to bounded domains. However to avoid difficulties we may consider only bounded, exterior and pipe-like domains with smooth boundaries.

At the boundary we impose a general boundary condition

$$
B(v, p)=0 \quad \text { on } \partial \Omega \times(0, T) .
$$

If the domain $\Omega$ is unbounded we add the condition at infinity

$$
v \rightarrow 0 \quad \text { as }|x| \rightarrow \infty .
$$

2000 Mathematics Subject Classification: 35Q30, 76D05.

Key words and phrases: global in time solutions, large data, stability, the Navier-Stokes equations, structure of initial data, regularity.

The paper is in final form and no version of it will be published elsewhere. 
To make our analysis concise we will restrict a class of general boundary conditions. The first restriction follows from the fact that we want to study regularity aspects of global in time weak solutions. Hence the basic energy estimate for solutions to system (1.1) is needed. It implies that properties of $B(\cdot, \cdot)$ must allow testing momentum equation $(1.1)_{1}$ with $v$ to get the energy inequality

$$
\frac{1}{2} \frac{d}{d t} \int_{\Omega} v^{2}+\nu \int_{\Omega}|\nabla v|^{2} d x \leq 0 .
$$

Assuming that there is no flow across the boundary we have

$$
v \cdot \vec{n}=0 \quad \text { on } \partial \Omega \times(0, T),
$$

where $\vec{n}$ is the normal vector to the boundary $\partial \Omega$. Also for sufficiently smooth solutions condition (1.2) has to imply that for a suitable class of divergence-free vectors,

$$
-\int_{\Omega} \Delta v v d x \geq C \int_{\Omega}|\nabla v|^{2} d x
$$

for a positive constant $C$. Then (1.5) and (1.6) guarantee inequality (1.4), provided sufficient regularity of solutions to (1.1) is assumed. Of course, in the general case these conditions can be replaced by weaker ones.

Moreover $B(v, p)=0$ have to satisfy the Lopatinskii-Shapiro condition [5] which imply that the system

$$
\begin{array}{lll}
-\nu \Delta v+\nabla p=f & \text { in } & \Omega, \\
\operatorname{div} v=0 & \text { in } & \Omega, \\
B(v, p)=0 & \text { on } & \partial \Omega
\end{array}
$$

should be elliptic well posed (in the case of unbounded domain we add (1.3)).

The standard example of relations $B(\cdot, \cdot)$ is the zero Dirichlet boundary conditions

$$
v \equiv 0 \quad \text { on } \partial \Omega \times(0, T),
$$

also known as the no-slip conditions. A natural generalization of (1.8) are slip boundary conditions [13], [14]

$$
\begin{gathered}
\vec{n} \cdot \mathbf{T}(v, p) \cdot \vec{\tau}_{k}+f v \cdot \vec{\tau}_{k}=0 \quad \text { on } \partial \Omega \times(0, T) \text { for } k=1,2 ; \\
v \cdot \vec{n}=0 \quad \text { on } \partial \Omega \times(0, T),
\end{gathered}
$$

where $\vec{\tau}_{k}$ are the tangent vectors to $\partial \Omega$. Relations (1.9) model friction effects by an introduction of the friction force described by the coefficient $f$ which generally may depend on the solution $(v, p)$, but from the physical point of view it should be nonnegative. Equation $(1.7)_{1}$ is just the second Newton principle. Note that as $f \rightarrow+\infty$, then condition (1.9) becomes (1.8), at least formally.

To simplify our consideration $B(\cdot, \cdot)$ in the proofs will be assumed to be a linear function. For nonlinear boundary models we refer to [6], [7]. If these relations are given by smooth functions and (1.4) and ellipticity of (1.7) are guaranteed, then the main result of our note stays true. However in that case our proof would be more complex, by adding unnecessarily difficult technical calculations. 
The basic information about solutions to the Navier-Stokes equations is given by the energy bound which is a consequence of inequality (1.4). The straightforward integration over time gives us the following result.

LEMMA 1.1. Let $v_{0} \in L_{2}(\Omega)$ and div $v_{0}=0$, then sufficiently smooth solutions to problem (1.1) defined on a time interval $(0, T)$ satisfy the energy estimate, i.e.

$$
\sup _{0 \leq t \leq T}\|v(\cdot, t)\|_{L_{2}(\Omega)} \leq\left\|v_{0}\right\|_{L_{2}(\Omega)} .
$$

An immediate corollary from Lemma 1.1 is that the $L_{2}$-norm of solutions will be arbitrarily small, provided smallness of the $L_{2}$-norm of the initial datum is assumed. As we see it will be the only quantity whose magnitude will be restricted in our considerations. Additionally by the Galerkin method this bound enables us to prove existence of weak solutions to (1.1).

The goal of this note is to prove global in time existence of regular solutions to the Navier-Stokes equations under a special assumptions of the structure of regularity of initial data $v_{0}$. Since the full solvability of this problem is still open we are able to prove only partial results - see [1], [2], [10], [13], [17].

We prove the following theorem which is the main result of the present paper.

Theorem 1.2. Let $1<p, q<\infty$ and $\frac{3}{p}+\frac{2}{q}<3$ and $v_{0} \in B_{p, q}^{2-2 / q}(\Omega) \cap L_{2}(\Omega)$. Suppose that

$$
\left\|v_{0}\right\|_{L_{2}(\Omega)} \text { is sufficiently small compared to }\left\|v_{0}\right\|_{B_{p, q}^{2-2 / q}(\Omega)} .
$$

Then there exists a unique global in time regular solution to the Navier-Stokes equations (1.1) such that $v \in W_{p, q(l o c)}^{2,1}(\Omega \times(0, \infty))$ and the following estimate holds

$$
\sup _{k \in \mathbf{N}}\|v\|_{W_{p, q}^{2,1}(\Omega \times[k, k+1])}<\infty .
$$

The above result yields solutions to the Navier-Stokes equations (1.1) in anisotropic Sobolev spaces $W_{p, q}^{2,1}$ - see the next section for necessary definitions. The main achievement is that the only restriction concerns the $L_{2}$-norm of initial datum $v_{0}$, the rest of the norm is not restricted. This extends the standard approach where the whole norm has to be controlled [2], [17]. The idea of the proof is based on an application of the energy estimate given by Lemma 1.1. We emphasize that this bound holds even for weak solutions. The energy bound neglects the influence of nonlinear transport term $v \cdot \nabla v$, thanks to that estimate (1.10) has a linear character and thanks to that information is possibly strongest. It is the only such estimate known for the Navier-Stokes equations for general cases - similar bounds can be found for restricted cases in two dimensions or in a symmetric case [12], [15], [16], [19]. Having Lemma 1.1 and suitable Schauder estimates for the Stokes system we relatively easily prove the main result using the theory of Sobolev-type spaces [4]. This approach has been effectively applied in stability problems - see [17], [20].

Comparing the presented approach to the technique based on application of the semigroup theory our method admits larger class of initial data. To apply tools of the theory of semigroups, first we have to project the system on the divergence free subspace. It follows that the nonlinear term looses its good structure and there is no chance to get 
a bound as (1.10). In the most optimal case we have to require smallness of the $L_{3}$-norm to obtain the global in time result [9]. And the regime of the projection makes the analysis more complex.

The obtained regularity of solutions enables, by classical results [18], to improve the regularity to any $C^{k}$-smoothness, under suitable conditions of initial data. Theorem 1.2 omits information about the pressure, since this sought function is completely determined by the velocity vector and estimates of $p$ do not play any role in our analysis.

Similar considerations have been done in [13], [14], however only for $W_{p, p}^{2,1}$-spaces for torus $\mathbf{T}^{3}$ and $\mathbf{R}^{3}$, domains without boundaries. Here we improve the method.

The paper is organized as follows. In section 2 we introduce basic notation and auxiliary results. Next, we show a sketch of the proof of the local in time existence result. In section 4 we prove Theorem 1.2. Generic constants are denoted by $C$.

2. Preliminaries. Here we introduce our notation and recall basic tools.

We try to follow the standard notation [11], [18]. The standard Lebesgue space $L_{p}(D)$ is the class of functions integrable with the $p$-th power over a set $D \subset \mathbf{R}^{d}$ with the norm

$$
\|u\|_{L_{p}(D)}=\left(\int_{D}|f(x)|^{p} d x\right)^{1 / p} \quad \text { for } 1 \leq p<\infty
$$

and

$$
\|u\|_{L_{\infty}(D)}=\underset{x \in D}{\operatorname{essipup}}|f(x)| \quad \text { for } p=\infty .
$$

Next, we define isotropic Besov spaces [4] $B_{p, q}^{s}(D)$ with $1 \leq p, q<\infty$ and $s \in \mathbf{R}_{+} \backslash \mathbf{N}$ by the following norm

$$
\|u\|_{B_{p, q}^{s}(D)}=\|u\|_{L_{p}(D)}+\langle u\rangle_{B_{p, q}^{s}(D)},
$$

where $\langle u\rangle_{B_{p, q}^{s}(D)}$ is the main seminorm of the $B_{p, q}^{s}$-space which reads

$$
=\sum_{|\alpha|=[s]}\left(\int_{B(0,1)} \frac{d h}{|h|^{d+s q}}\left(\int_{(D+h) \cap D}\left|\partial_{x}^{\alpha} u(x+h)-\partial_{x}^{\alpha} u(x)\right|^{p} d x\right)^{q / p}\right)^{1 / q},
$$

where $\alpha=\left(\alpha_{1}, \ldots, \alpha_{d}\right)$ is the multi-index, $|\alpha|=\alpha_{1}+\cdots+\alpha_{d},[\cdot]$ is the integral part of number $s, B(0,1)$ is the unit ball in $\mathbf{R}^{d}$ with center at the origin. Set $D+h=\left\{z \in \mathbf{R}^{d}\right.$ : $z=x+h$ and $x \in D\}$.

We restrict out attention to cases where parameter $s$ is not a natural number, because we will work with this case only. The defined Besov class describes the trace space for the anisotropic Sobolev space, but first let us recall the standard Sobolev space $W_{p}^{m}(D)$ defined by the norm

$$
\|u\|_{W_{p}^{m}(D)}=\|u\|_{L_{p}(D)}+\sum_{|\alpha|=m}\left\|\partial_{x}^{\alpha} u\right\|_{L_{p}(D)}
$$

for $m \in \mathbf{N}$ and $1 \leq p<\infty$.

Unfortunately, isotropic spaces are not sufficient in our method, because of the evolutionary character of the system. There is a need for a class of spaces which distinguish 
the time direction. For functions defined on $D \times(0, T)$ we introduce the $W_{p, q}^{m, n}(D \times(0, T))$ space for $m, n \in \mathbf{N}$ and $1 \leq p, q<\infty$ by the norm

$$
\begin{gathered}
\|u\|_{W_{p, q}^{m, n}(D \times(0, T))}=\|u\|_{L_{q}\left(0, T ; L_{p}(D)\right)} \\
+\sum_{|\alpha|=m}\left(\int_{0}^{T}\left(\int_{D}\left|\partial_{x}^{\alpha} u\right|^{p} d x\right)^{q / p} d t\right)^{1 / q}+\left(\int_{0}^{T}\left(\int_{D}\left|\partial_{t}^{n} u\right|^{p} d x\right)^{q / p} d t\right)^{1 / q} .
\end{gathered}
$$

The above norms have a natural generalization for $p=\infty$ or $q=\infty$ - see (2.1) and (2.2).

The definition of the Besov spaces $B_{p, q}^{s}$ can be stated by using interpolation theory [3]. For $s \in(0,1)$ we have

$$
B_{p, q}^{s}(\Omega)=\left(L_{p}(\Omega), W_{p}^{1}(\Omega)\right)_{s, q} .
$$

The theory of Besov spaces yields the following imbedding theorem:

Proposition 2.1. Let $m, n>0,1 \leq p \leq k \leq \infty$ and $1 \leq q \leq l \leq \infty$. If

$$
\frac{d}{m}\left(\frac{1}{p}-\frac{1}{k}\right)+\frac{1}{n}\left(\frac{1}{q}-\frac{1}{l}\right)<1,
$$

then $W_{p, q}^{m, n}(D \times(0, T)) \subset L_{l}\left(0, T ; L_{k}(D)\right)$, moreover there exists a function $I(\cdot)$ such that $I(\epsilon) \rightarrow \infty$ as $\epsilon \rightarrow 0$ and

$$
\|u\|_{L_{l}\left(0, T ; L_{k}(D)\right)} \leq \epsilon\langle u\rangle_{W_{p, q}^{m, n}(D \times(0, T))}+I(\epsilon)\|u\|_{L_{2}\left(0, T ; L_{2}(D)\right)} .
$$

Next, we recall the trace theorem for $W_{p, q}^{2,1}$-spaces.

Proposition 2.2. Let $1 \leq p, q<\infty$ then $\left.\operatorname{Tr}\right|_{t=0} W_{p, q}^{2,1}(D \times(0, T)) \subset B_{p, q}^{2-2 / q}(D)$, i.e. if $u \in W_{p, q}^{2,1}(D \times(0, T))$, then $\left.u\right|_{t=0} \in B_{p, q}^{2-2 / q}(D)$ and

$$
\left\|\left.u\right|_{t=0}\right\|_{B_{p, q}^{2-2 / q}(D)} \leq C\|u\|_{W_{p, q}^{2,1}(D \times(0, T))} .
$$

A key element of our technique is the Schauder estimate of solutions to the Stokes system which is a linearization of nonlinear equations (1.1)

$$
\begin{array}{ll}
u_{t}-\nu \Delta u+\nabla q=f & \text { in } \quad \Omega \times(0, T), \\
\operatorname{div} u=0 & \text { in } \quad \Omega \times(0, T), \\
B(u, p)=0 & \text { on } \quad \partial \times(0, T), \\
\left.u\right|_{t=0}=u_{0} & \text { on } \quad \Omega,
\end{array}
$$

where data satisfy suitable compatibility conditions. The classical theory - see [8] and [3] - guarantees us the following existence result in optimal spaces obtained by the advanced theory of semigroups.

Propositions 2.3. Let $u_{0} \in B_{p, q}^{2-2 / q}(\Omega), f \in L_{q}\left(0, T ; L_{p}(\Omega)\right)$ and div $u_{0}=0$, then there exists a unique solution to system (2.9) such that

$$
u \in W_{p, q}^{2,1}(\Omega \times(0, T)) \quad \text { and } \quad \nabla q \in L_{q}\left(0, T ; L_{p}(\Omega)\right),
$$

moreover the following estimate is valid

$$
\begin{gathered}
\left\|u_{t}\right\|_{L_{q}\left(0, T ; L_{p}(\Omega)\right)}+\left\|\nabla^{2} u\right\|_{L_{q}\left(0, T ; L_{p}(\Omega)\right)}+\|\nabla q\|_{L_{q}\left(0, T ; L_{p}(\Omega)\right)} \\
\leq A\left(\|f\|_{L_{q}\left(0, T ; L_{p}(\Omega)\right)}+\left\|u_{0}\right\|_{\left.B_{p, q}^{2-2 / q}(\Omega)\right)}\right)
\end{gathered}
$$

and the constant $A$ is independent of $T$, in particular we can consider $T=\infty$. 
3. Local existence. In this section we show the local in time existence of solutions to system (1.1). Thanks to this result the proof of Theorem 1.2 will be reduced to a suitable a priori estimate. Then by the trace theorem - Proposition 2.2 - we will be able to prolong the solution to the time interval where the a priori estimate is valid.

THEOREM 3.1. Let $p, q$ satisfy $\frac{3}{p}+\frac{2}{q}<3, v_{0} \in B_{p, q}^{2-2 / q}(\Omega)$ and div $v_{0}=0$, then there exists a number $T_{0}>0$ such that there exists a unique solution to system (1.1) on the time interval $[0, T]$ for $T \leq T_{0}$ in the following class of regularity

$$
v \in W_{p, q}^{2,1}(\Omega \times(0, T)), \quad p \in W_{p, q}^{1,0}(\Omega \times(0, T)) ;
$$

moreover the following estimate is valid

$$
\|v\|_{W_{p, q}^{2,1}(\Omega \times(0, T))}+\|\nabla p\|_{W_{p, q}^{1,0}(\Omega \times(0, T))} \leq C\left(\left\|v_{0}\right\|_{B_{p, q}^{2-2 / q}(\Omega)}\right) .
$$

Thus $T_{0}$ depends on the magnitude of the norm $\left\|v_{0}\right\|_{B_{p, q}^{2-2 / q}(\Omega)}$, only.

Proof. We present only a sketch of the proof. It is based on the standard application of the Banach fixed point theorem to a suitable linearization. In our case it is just the Stokes system. (For details we refer to [14] for consideration for Sobolev spaces with case $p=q)$.

The only difficulty in the proof of Theorem 3.1 is the nonlinear term $v \cdot \nabla v$ which by Proposition 2.1 has to be bounded in $L_{q}\left(0, T ; L_{p}(\Omega)\right)$.

By the imbedding theorem - Proposition 2.1 - we have

$$
W_{p, q}^{2,1}(\Omega \times(0, T)) \subset L_{b_{1}}\left(0, T ; L_{a_{1}}(\Omega)\right),
$$

provided $\frac{3}{2}\left(\frac{1}{p}-\frac{1}{a_{1}}\right)+\left(\frac{1}{q}-\frac{1}{b_{1}}\right)<1$ and

$$
\nabla W_{p, q}^{2,1}(\Omega \times(0, T)) \subset L_{b_{2}}\left(0, T ; L_{a_{2}}(\Omega)\right),
$$

provided $\frac{3}{2}\left(\frac{1}{p}-\frac{1}{a_{2}}\right)+\left(\frac{1}{q}-\frac{1}{b_{2}}\right)<\frac{1}{2}$. Applying (3.2) and (3.3) we get

$$
\|v \cdot \nabla v\|_{L_{q}\left(0, T ; L_{p}(\Omega)\right)} \leq C\|v\|_{L_{b_{1}}\left(0, T ; L_{a_{1}}(\Omega)\right)}\|\nabla v\|_{L_{b_{2}}\left(0, T ; L_{a_{2}}(\Omega)\right)},
$$

if we assume $\frac{1}{p}=\frac{1}{a_{1}}+\frac{1}{a_{2}}$ and $\frac{1}{q}=\frac{1}{b_{1}}+\frac{1}{b_{2}}$. The last restriction can be fulfilled only if

$$
\frac{3}{p}+\frac{2}{q}<3
$$

The above bound on the powers $p$ and $q$ is connected with the Serrin condition [18] guaranteeing regularity of weak solutions. By Proposition 2.1 and (3.5) we can find $l$ and $m$ such that

$$
W_{p, q}^{2,1}(\Omega \times(0, T)) \subset L_{m}\left(0, T ; L_{l}(\Omega)\right) \quad \text { with } \quad \frac{3}{l}+\frac{2}{m}<1,
$$

and the last inequality in (3.6) is the original restriction given by Serrin.

For simplicity we may put in (3.4) factors $a_{1}=3 p, b_{1}=3 q, a_{2}=\frac{3}{2} p$ and $b_{2}=\frac{3}{2} q$.

An important question is dependence of constant $C$ in (3.4) on the smallness of $T$. Thanks to standard tricks - see [13] - and the fact that in (3.5) the inequality is strict, we are able to get for $0<T<1$ the following estimate

$$
\|v \cdot \nabla v\|_{L_{q}\left(0, T ; L_{p}(\Omega)\right)} \leq C_{0} T^{\alpha}\|v\|_{W_{p, q}^{2,1}(\Omega \times(0, T))}^{2}
$$

for a number $\alpha>0$ and $c_{0}$ independent of $T$. 
Inequality (3.7) allows us to find the solution to (1.1) as the fixed point to a map $K$ such that

$$
K: W_{p, q}^{2,1}(\Omega \times(0, T)) \rightarrow W_{p, q}^{2,1}(\Omega \times(0, T)) \quad \text { and } \quad K(\tilde{v})=v,
$$

where $v$ is defined as a solution to the following problem

$$
\begin{array}{ll}
v_{t}-\nu \Delta v+\nabla p=-\tilde{v} \cdot \nabla \tilde{v} & \text { in } \Omega \times(0, T), \\
\operatorname{div} v=0 & \text { in } \Omega \times(0, T), \\
B(u, p)=0 & \text { on } \partial \Omega \times(0, T), \\
\left.v\right|_{t=0}=v_{0} & \text { on } \Omega .
\end{array}
$$

By Proposition 2.3 we immediately get the existence of solutions to system (3.8) and the following bound on $v$

$$
\|v\|_{W_{p, q}^{2,1}(\Omega \times(0, T))} \leq C\left(\|\tilde{v} \cdot \nabla \tilde{v}\|_{L_{q}\left(0, T ; L_{p}(\Omega)\right)}+\left\|v_{0}\right\|_{B_{p, q}^{2-2 / q}(\Omega)}\right) .
$$

Employing inequality (3.7) we obtain

$$
\|v\|_{W_{p, q}^{2,1}(\Omega \times(0, T))} \leq A T^{\alpha}\|\tilde{v}\|_{W_{p, q}^{2,1}(\Omega \times(0, T))}^{2}+B\left\|v_{0}\right\|_{B_{p, q}^{2-2 / q}(\Omega)}
$$

for some fixed positive constants $A$ and $B$.

The above inequality guarantees that

$$
\|v\|_{W_{p, q}^{2,1}(\Omega \times(0, T))} \leq 2 B\left\|v_{0}\right\|_{B_{p, q}^{2-2 / q}(\Omega)},
$$

provided

$$
\|\tilde{v}\|_{W_{p, q}^{2,1}(\Omega \times(0, T))} \leq 2 B\left\|v_{0}\right\|_{B_{p, q}^{2-2 / q}(\Omega)},
$$

if $T>0$ is so small that $4 A B T^{\alpha}\left\|v_{0}\right\|_{B_{p, q}^{2-2 / q}(\Omega)} \leq 1$.

Thus $K$ maps a bounded ball in $W_{p, q}^{2,1}$ into itself. Next, we shall show that $K$ truncated to this set is a contraction. We skip this part of the proof, since it is almost the same as considerations for $(3.10)$ - possibly with smaller $T$. Thus there exists a fixed point of $K$ defining the sought solutions to system (1.1) in the desired class of regularity. Thus Theorem 3.1 is proved.

4. Global existence. For given initial data in $B_{p, q}^{2-2 / q}(\Omega)$ and Theorem 3.1 we are able to find a number $T_{0}>0$ such that the solution to system (1.1) exists in time interval $\left[0, T_{0}\right]$ with a bound given by $(3.1)$.

For a number $L<T_{0}$ we construct a sequence of smooth functions $\zeta^{k}: \mathbf{R}_{+} \rightarrow[0,1]$ such that

$$
\zeta^{k}(t)= \begin{cases}1 & \text { for } t \geq k L, \\ \in[0,1] & \text { for } \quad(k-1) L \leq t \leq k L, \\ 0 & \text { for } t \leq(k-1) L\end{cases}
$$

and $k \in \mathbf{N}$ with $\left|\zeta^{\prime}(t)\right| \leq \frac{2}{L}$.

Functions $\zeta^{k}(\cdot)$ are auxiliary tools to correct sought solution $(v, p)$ of original system (1.1). We define

$$
V_{k}(x, t)=\zeta^{k}(t) v(x, y) \quad \text { and } \quad P_{k}(x, t)=\zeta^{k}(t) p(x, t),
$$

then we consider a system being modification of the original equations, but only for time interval $t \in[(k-1) L,(k+1) L]$. By Theorem 3.1 we know that there exists a maximal time $T_{\max }$ for which solutions to system (1.1) exist. To avoid formal analysis we can 
assume that $k$ in (4.2) is taken only for $k \leq\left[T_{\max } / L\right]$ and we ask if the solution can be prolong on $\left[0, T_{\max }+L\right]$.

From (1.1) and (4.2) we have

$$
\begin{array}{ll}
V_{k, t}-\nu \Delta V_{k}+\nabla P_{k}=v \cdot \nabla V_{k}+\zeta_{k}^{\prime} v & \text { in } \Omega \times((k-1) L,(k+1) L), \\
\operatorname{div} V_{k}=0 & \text { in } \Omega \times((k-1) L,(k+1) L), \\
B\left(V_{k}, P_{k}\right)=0 & \text { on } \partial \Omega \times((k-1) L,(k+1) L), \\
\left.V_{k}\right|_{t=(k-1) L}=0 & \text { on } \Omega .
\end{array}
$$

Now, we are ready to apply Proposition 2.3 to system (4.3) getting the estimate on $V_{k}$ and $P_{k}$

$$
\begin{gathered}
\left\|V_{k}\right\|_{W_{p, q}^{2,1}(\Omega \times((k-1) L,(k+1) L))}+\left\|\nabla P_{k}\right\|_{L_{q}\left((k-1) L,(k+1) L ; L_{p}(\Omega)\right)} \\
\leq C\left(\left\|v \cdot \nabla V_{k}\right\|_{L_{q}\left((k-1) L,(k+1) L ; L_{p}(\Omega)\right)}+\left\|\zeta^{\prime} v\right\|_{L_{q}\left((k-1) L,(k+1) L ; L_{p}(\Omega)\right)}\right) .
\end{gathered}
$$

We consider the r.h.s. of inequality (4.4).

For simplicity let us introduce the following quantity

$$
\Xi_{k}=\left\|V_{k}\right\|_{W_{p, q}^{2,1}(\Omega \times((k-1) L,(k+1) L))} \cdot
$$

Applying the imbedding theorem - Proposition 2.1 - we estimate terms of the r.h.s. of (4.4). For small $\epsilon>0$ the last term if the r.h.s. of (4.4) is bounded as follows

$$
\left\|\zeta^{\prime} v\right\|_{L_{q}\left((k-1) L,(k+1) L ; L_{p}(\Omega)\right)} \leq \frac{2}{L}\left(\epsilon \Xi_{k-1}+I(\epsilon)\|v\|_{L_{\infty}\left((k-1) L,(k+1) L ; L_{2}(\Omega)\right)}\right) .
$$

Next, we examine the nonlinear term in two parts related to two time intervals: $((k-1) L, k L)$ and $(k L ;(k+1) L)$. We have

$$
\begin{gathered}
\left\|v \cdot \nabla V_{k}\right\|_{L_{q}\left((k-1) L,(k+1) L ; L_{p}(\Omega)\right)} \leq \\
\left\|V_{k-1} \cdot \nabla V_{k}\right\|_{L_{q}\left((k-1) L, k L ; L_{p}(\Omega)\right)}+\left\|V_{k} \cdot \nabla V_{k}\right\|_{L_{q}\left(k L,(k+1) L ; L_{p}(\Omega)\right)} .
\end{gathered}
$$

To estimate the first term of the r.h.s. of (4.7) we use the fact that in imbedding conditions we have a strict inequality, thus there is room for interpolation. So

$$
\begin{gathered}
\left\|V_{k-1} \cdot \nabla V_{k}\right\|_{L_{q}\left((k-1) L, k L ; L_{p}(\Omega)\right)} \\
\leq\left\|V_{k-1}\right\|_{L_{b_{1}}\left((k-1) L, k L ; L_{a_{1}}(\Omega)\right)}\left\|\nabla V_{k}\right\|_{L_{b_{2}}\left(k L,(k+1) L ; L_{a_{2}}(\Omega)\right)}
\end{gathered}
$$

and from (2.7) we get ( $L$ is fixed)

$$
\begin{aligned}
\left\|V_{k-1}\right\|_{L_{b_{1}}\left((k-1) L, k L ; L_{a_{1}}(\Omega)\right)} & \leq \epsilon \Xi_{k-1}+I(\epsilon)\|v\|_{L_{\infty}\left(0, T_{\max } ; L_{2}(\Omega)\right)}, \\
\left\|\nabla V_{k}\right\|_{L_{b_{2}}\left(k L,(k+1) L ; L_{a_{2}}(\Omega)\right)} & \leq \epsilon \Xi_{k}+I(\epsilon)\|v\|_{L_{\infty}\left(0, T_{\max } ; L_{2}(\Omega)\right)} .
\end{aligned}
$$

Thus by (4.8)-(4.10) the first term of the r.h.s. of (4.7) is bounded by

$$
\left\|V_{k-1} \cdot \nabla V_{k}\right\|_{L_{q}\left((k-1) L, k L ; L_{p}(\Omega)\right)} \leq \epsilon^{2} \Xi_{k}^{2}+\epsilon^{2} \Xi_{k-1}^{2}+I(\epsilon)^{2}\|v\|_{L_{\infty}\left(0, T_{\max } ; L_{2}(\Omega)\right)}^{2} .
$$

In the same way we get the bound for the second term of (4.7)

$$
\left\|V_{k} \cdot \nabla V_{k}\right\|_{L_{q}\left((k-1) L, k L ; L_{p}(\Omega)\right)} \leq \epsilon^{2} \Xi_{k}^{2}+I(\epsilon)^{2}\|v\|_{L_{\infty}\left(0, T_{\max } ; L_{2}(\Omega)\right)} .
$$

The r.h.s. of (4.12) is immediately bounded from Lemma 1.1 by the $L_{2}$-norm of initial datum $v_{0}$. Thus from (4.4), (4.6) and (4.12) we conclude

$$
\Xi_{k} \leq \epsilon^{2} \Xi_{k}^{2}+\epsilon^{2} \Xi_{k-1}^{2}+I(\epsilon)^{2}\left\|v_{0}\right\|_{L_{2}(\Omega)}^{2}
$$


for a chosen small $\epsilon>0$. We want to show by induction that for any $k \in \mathbf{N}$

$$
\Xi_{k} \leq M, \quad \text { provided } \quad M \leq 2 B\left\|v_{0}\right\|_{B_{p, q}^{2-2 / q}(\Omega)} .
$$

By Theorem 3.1 condition (4.14) for $k=0$ holds. Assuming that bound (4.14) holds for $k-1$ we want to show it for $k$. From (4.13) we conclude

$$
\Xi_{k} \leq \epsilon^{2} \Xi_{k}^{2}+\epsilon^{2} M^{2}+I(\epsilon)^{2}\left\|v_{0}\right\|_{L_{2}(\Omega)}^{2} .
$$

The above inequality implies estimate (4.14) if

$$
\frac{1}{2 \epsilon^{2}}\left(1-\sqrt{1-4 \epsilon^{2}\left(\epsilon^{2} M^{2}+I(\epsilon)^{2}\left\|v_{0}\right\|_{L_{2}(\Omega)}^{2}\right.}\right) \leq M .
$$

To fulfill condition (4.16) we choose so small $\epsilon>0$ that

$$
\epsilon^{4} M^{2}<\frac{1}{8}
$$

For chosen $\epsilon$ we prescribe the restriction on the $L_{2}$-norm of the initial datum: assume that

$$
\epsilon^{2} I(\epsilon)^{2}\left\|v_{0}\right\|_{L_{2}(\Omega)}^{2}<\frac{1}{8} .
$$

Then the l.h.s. of (4.16) is well defined as a real number and by (4.17) and (4.18) we have

$$
\frac{1}{2 \epsilon^{2}}\left(1-\sqrt{1-4 \epsilon^{2}\left(\epsilon^{2} M^{2}+I(\epsilon)^{2}\left\|v_{0}\right\|_{L_{2}(\Omega)}^{2}\right)} \leq \frac{1}{4} M+I(\epsilon)^{2}\left\|v_{0}\right\|_{L_{2}(\Omega)}^{2} .\right.
$$

As $\epsilon$ is fixed thus $I(\epsilon)$, too, hence the suitable smallness of the $L_{2}$-norm implies immediately that the r.h.s. of (4.19) is less than $M$. This shows statement (4.13). By definition (4.5) and finiteness of $L$ we conclude estimate (1.12) from Theorem 1.2. Then the trace theorem and the local in time existence - Theorem 3.1 - guarantees global in time existence of solutions. Theorem 1.2 is proved.

Acknowledgments. The work has been supported by Polish KBN grant No. 1 P03A 02130 and by ECFP6 M.Curie ToK program SPADE2, MTKD-CT-2004-014508 and SPB-M.

\section{References}

[1] A. Babin, A. Mahalov and B. Nicolaenko, Global regularity of 3D rotating Navier-Stokes equations for resonant domains, Indiana Univ. Math. J. 48 (1999), 1133-1176.

[2] H. Beirão da Veiga and P. Secchi, $L^{p}$-stability for the strong solutions of the Navier-Stokes equations in the whole space, Arch. Rational Mech. Anal. 98 (1987), 65-69.

[3] C. Bennett and R. Sharpley, Interpolation of Operators, Pure and Applied Mathematics 129, Academic Press, Inc., Boston, MA, 1988.

[4] O. V. Besov, V. P. Ilin and S. M. Nikolskij, Integral Representation of Functions and Imbedding Theorems, Moscow, 1975.

[5] R. Denk, M. Hieber and J. Prüss, R-boundedness, Fourier multipliers and problems of elliptic and parabolic type, Mem. Amer. Math. Soc. 166 (2003), no. 788.

[6] H. Fujita, Remarks on the Stokes flow under slip and leak boundary conditions of friction type, in: Topics in Mathematical Fluid Mechanics, Quad. Mat. 10, 2002, 73-94. 
[7] S. Itoh, N. Tanaka and A. Tani, The initial value problem for the Navier-Stokes equations with general slip boundary condition, Adv. Math. Sci. Appl. 4 (1994), 51-69.

[8] Y. Giga and H. Sohr, Abstract $L^{p}$ estimates for the Cauchy problem with applications to the Navier-Stokes equations in exterior domains, J. Funct. Anal. 102 (1991), 72-94.

[9] T. Kato, Strong $L^{p}$-solutions of the Navier-Stokes equations in $R^{m}$, with application to weak solutions, Math. Z. 187 (1984), 471-480.

[10] O. A. Ladyzhenskaya, Solution "in the large" of the non-stationary boundary value problem for the Navier-Stokes system with two space variables, Comm. Pure Appl. Math. 12 (1959), 427-433.

[11] O. A. Ladyzhenskaya, The Mathematical Theory of Viscous Incompressible Flow, Gordon and Breach, New York, 1969.

[12] O. A. Ladyzhenskaya, Unique global solvability of the three-dimensional Cauchy problem for the Navier-Stokes equations in the presence of axial symmetry, Zap. Naučn. Sem. Leningrad. Otdel. Mat. Inst. Steklov. (LOMI) 7 (1968), 155-177 (in Russian).

[13] P. B. Mucha, Stability of nontrivial solutions of the Navier-Stokes system on the three dimensional torus, J. Differential Equations 172 (2001), 359-375.

[14] P. B. Mucha, Stability of constant solutions to the Navier-Stokes system in $\mathbf{R}^{3}$, Appl. Math. (Warsaw) 28 (2001), 301-310.

[15] P. B. Mucha, The Navier-Stokes equations and the maximum principle, Int. Math. Res. Not. 2004, no. 67, 3585-3605.

[16] P. B. Mucha, On the structure of flows through pipe-like domains satisfying a geometrical constraint, Appl. Math. (Warsaw) 31 (2004), 457-471.

[17] G. Ponce, R. Racke, T. C. Sideris, and E. S. Titi, Global stability of large solutions to the 3D Navier-Stokes equations, Comm. Math. Phys. 159 (1994), 329-341.

[18] H. Sohr, The Navier-Stokes Equations. An Elementary Functional Analytic Approach, Birkhäuser Verlag, Basel, 2001.

[19] M. R. Ukhovskii and V. I. Yudovich, Axially symmetric flows of ideal and viscous fluids filling the whole space, Prikl. Mat. Meh. 32 59-69 (in Russian); English transl.: J. Appl. Math. Mech. 32 (1968), 52-61.

[20] W. M. Zajączkowski, Global special regular solutions to the Navier-Stokes equations in a cylindrical domain under boundary slip conditions, Gakuto Series in Math. 21 (2004). 